\title{
Eccentric Domination and Restrained Eccentric Domination in Circulant Graphs
}

\author{
M. Bhanumathi ${ }^{1}$ and ${ }^{*}$ R. Niroja ${ }^{2}$ \\ ${ }^{1}$ Government Arts and Science College, Kadaladi, Ramanathapuram, Tamilnadu, India. \\ ${ }^{2}$ Government Arts College for Women (Autonomous), Pudukkottai-622001, Tamilnadu, India. \\ EMail: bhanu_ksp@yahoo.com,*nironeela@gmail.com
}

\begin{abstract}
A$ subset $D$ of the vertex set $V(G)$ of a graph $G$ is said to be a dominating set if every vertex not in $D$ is adjacent to at least one vertex in $D$. A dominating set $D$ is said to be an eccentric dominating set if for every $v \in V-D$, there exists at least one eccentric vertex of $v$ in $D$. Let $p \geq 4$ be a positive integer. The circulant graph $C_{p}\langle 1,2\rangle$ is the graph with vertex set $\left\{v_{0}, v_{1}, v_{2}, \ldots, v_{p-1}\right\}$ and edge set $\left\{\left\{v_{i}, v_{i+j}\right\}: i \in\{0,1,2, \ldots, p-1\}\right.$ and $\left.j \in\{1,2\}\right\}$. In this paper, we initiate the study of domination number, eccentric domination number and restrained eccentric domination number in the circulant graphs $C_{p}\langle 1,2\rangle$.
\end{abstract}

Keywords: domination, eccentric domination, restrained eccentric domination, circulant graphs. Mathematics Subject Classification: 05C12, 05C69.

\section{Introduction}

Let $\mathrm{G}$ be a finite, simple, undirected $(\mathrm{p}, \mathrm{q})$ graph with vertex set $\mathrm{V}(\mathrm{G})$ and edge set $\mathrm{E}(\mathrm{G})$. For graph theoretic terminology refer to Harary [5], Buckley and Harary [3].

The concept of domination in graphs is originated from the chess games theory and that paved the way to the development of the study of various domination parameters and its relation to various other graph parameters. For details on domination theory, refer to Haynes, Hedetniemi and Slater [8]. Janakiraman, Bhanumathi and Muthammai [6] introduced Eccentric domination in Graphs. Bhanumathi, John Flavia and Kavitha [1] introduced and studied the concept of Restrained Eccentric domination in Graphs.

Definition 1.1: Let $\mathrm{p} \geq 4$ be a positive integer. The circulant graph $\mathrm{C}_{\mathrm{p}}\langle 1,2\rangle$ is the graph with vertex set $\left\{\mathrm{v}_{0}, \mathrm{v}_{1}, \mathrm{v}_{2}, \ldots, \mathrm{v}_{\mathrm{p}-1}\right\}$ and edge set $\left\{\left\{\mathrm{v}_{\mathrm{i}}, \mathrm{v}_{\mathrm{i}+\mathrm{j}}\right\}: \mathrm{i} \in\{0,1,2, \ldots, \mathrm{p}-1\}\right.$ and $\left.\mathrm{j} \in\{1,2\}\right\}$.

Definition 1.2: Let $G$ be a connected graph and $v$ be a vertex of $G$. The eccentricity $e(v)$ of $\mathrm{v}$ is the distance to a vertex farthest from $\mathrm{v}$. Thus, $\mathrm{e}(\mathrm{v})=\max \{\mathrm{d}(\mathrm{u}, \mathrm{v}): \mathrm{u} \in \mathrm{V}\}$. The radius $r(G)$ is the minimum eccentricity of the vertices, whereas the diameter $\operatorname{diam}(G)=d(G)$ is the maximum eccentricity. For any connected graph $G, r(G) \leq \operatorname{diam}(G) \leq 2 r(G)$. The vertex $v$ is a central vertex if $e(v)=r(G)$. The center $C(G)$ is the set of all central vertices.

For a vertex $v$, each vertex at a distance $e(v)$ from $v$ is an eccentric vertex of $v$. Eccentric set of a vertex $\mathrm{v}$ is defined as $\mathrm{E}(\mathrm{v})=\{\mathrm{u} \in \mathrm{V}(\mathrm{G}) / \mathrm{d}(\mathrm{u}, \mathrm{v})=\mathrm{e}(\mathrm{v})\}$. 
Definition 1.3: A graph $G$ is called a m-eccentric point graph if each point of $G$ has exactly $\mathrm{m} \geq 1$ eccentric points.

Definition 1.4 [4, 8]: A set $\mathrm{D} \subseteq \mathrm{V}$ is said to be a dominating set in $\mathrm{G}$, if every vertex in $\mathrm{V}-\mathrm{D}$ is adjacent to some vertex in $\mathrm{D}$. The minimum cardinality of a dominating set is called the domination number and is denoted by $\gamma(G)$.

Definition 1.5 [5]: A set $\mathrm{D} \subseteq \mathrm{V}(\mathrm{G})$ is a restrained dominating set if every vertex not in $\mathrm{D}$ is adjacent to a vertex in $\mathrm{D}$ and to a vertex in $\mathrm{V}-\mathrm{D}$. The cardinality of minimum restrained dominating set is called the restrained domination number and is denoted by $\gamma_{\mathrm{r}}(\mathrm{G})$

Definition 1.6 [6]: A set $\mathrm{D} \subseteq \mathrm{V}(\mathrm{G})$ is an eccentric dominating set if $\mathrm{D}$ is a dominating set of $G$ and for every $v \in V-D$, there exists at least one eccentric vertex of $v$ in $D$. The minimum cardinality of an eccentric dominating set is called the eccentric domination number and is denoted by $\gamma_{\text {ed }}(G)$.

Definition 1.7 [1]: A subset $D$ of $V(G)$ is a restrained eccentric dominating set if $D$ is a restrained dominating set of $\mathrm{G}$ and for every $\mathrm{v} \in \mathrm{V}-\mathrm{D}$, there exists at least one eccentric vertex of $\mathrm{v}$ in $\mathrm{D}$. The minimum of the cardinalities of the restrained eccentric dominating set of $G$ is called the restrained eccentric domination number of $G$ and is denoted by $\gamma_{\text {red }}(G)$.

Theorem 1.1 [8]: For any graph $G,[p /(1+\Delta(G))] \leq \gamma(G) \leq p-\Delta(G)$.

\section{Domination, Eccentric Domination, Restrained Domination and Restrained Eccentric Domination.}

In this section, we determine the eccentric domination and the restrained eccentric domination number of circulant graphs $\quad G=C_{p}\langle 1,2\rangle$, for any integer $p \geq 4$. Cleary, $\mathrm{G}$ is a 4-regular graph on p vertices.

It is obvious that $C_{4}\langle 1,2\rangle=K_{4}$ and $C_{5}\langle 1,2\rangle=K_{5}$. So $\quad \gamma\left(C_{4}\langle 1,2\rangle\right)=\gamma_{\text {ed }}\left(C_{4}\langle 1\right.$, $2\rangle)=\gamma_{\mathrm{r}}\left(\mathrm{C}_{4}\langle 1,2\rangle\right)=\gamma_{\text {red }}\left(\mathrm{C}_{4}\langle 1,2\rangle\right)=1$ and $\gamma\left(\mathrm{C}_{5}\langle 1,2\rangle\right)=\gamma_{\mathrm{ed}}\left(\mathrm{C}_{5}\langle 1,2\rangle\right)=\gamma_{\mathrm{r}}\left(\mathrm{C}_{5}\langle 1,2\rangle\right)=$ $\gamma_{\text {red }}\left(C_{5}\langle 1,2\rangle\right)=1$. For $\quad \mathrm{p} \geq 6$ we have the following results: 
Example: 2.1

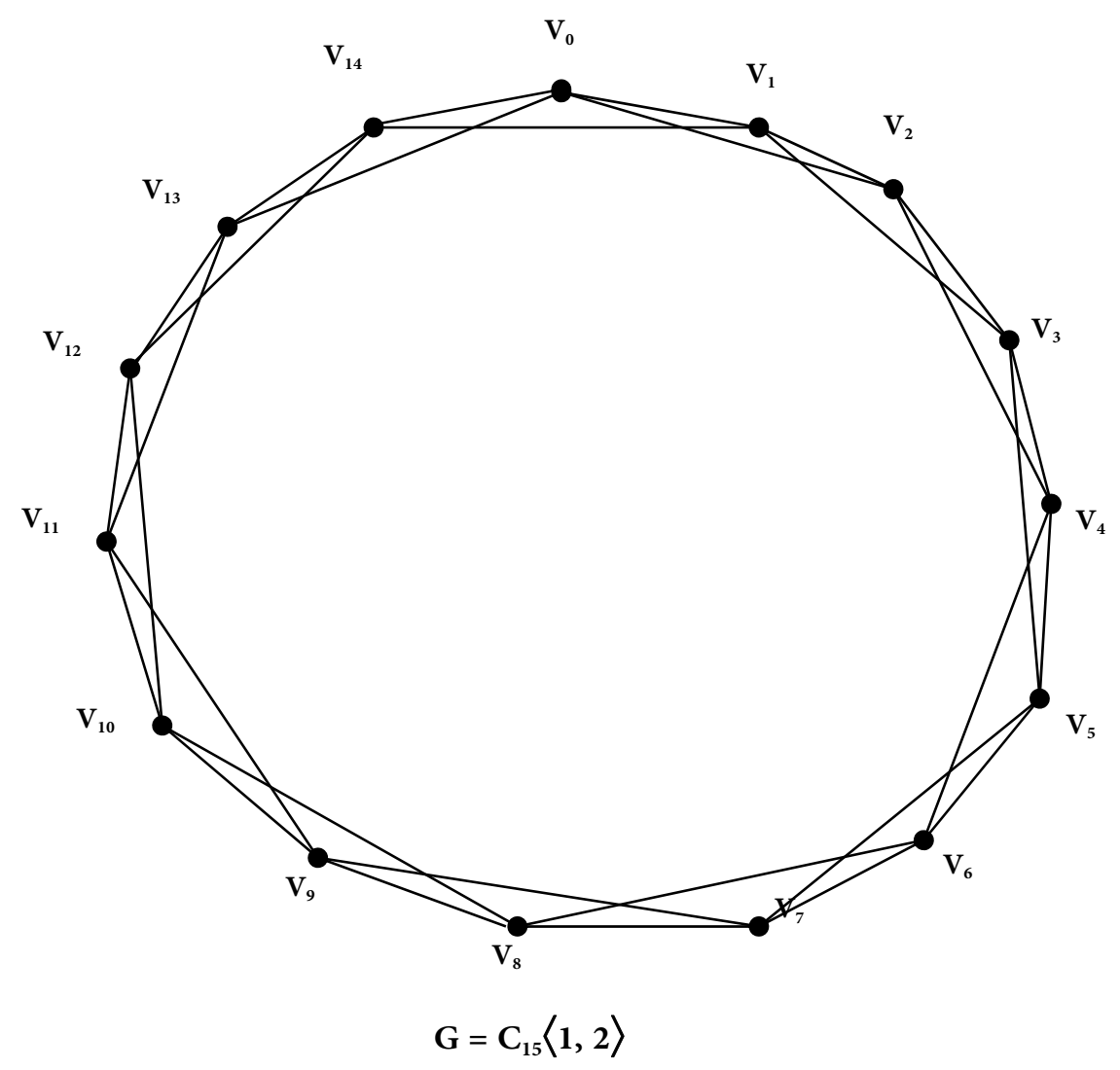

Figure 2.1

In Figure 2.1,

$\mathrm{D}_{1}=\left\{\mathrm{v}_{0}, \mathrm{v}_{5}, \mathrm{v}_{10}\right\}$ is a dominating set of $\mathrm{G}$ and is also a restrained dominating set of $\mathrm{G}$.

Therefore, $\gamma(\mathrm{G})=\gamma_{\mathrm{r}}(\mathrm{G})=3$.

$D_{2}=\left\{v_{0}, v_{3}, v_{6}, v_{9}, v_{12}\right\}$ is an eccentric dominating set of $G$ and is also a restrained eccentric dominating set of $G$. Therefore, $\gamma_{\text {ed }}(G)=\gamma_{\text {red }}(G)=5$.

Lemma 2.1: Let $G=C_{p}\langle 1,2\rangle$ be a connected graph then for any integer $p \geq 6, \gamma(G)=$ $\gamma_{\mathrm{r}}(\mathrm{G})=\lceil\mathrm{p} / 5\rceil$.

Proof: Let $G=C_{p}\langle 1,2\rangle$ and let $v_{0}, v_{1}, v_{2}, \ldots, v_{p-1}$ be the vertices of G. From Theorem 1.1, $\lceil\mathrm{p} /(1+\Delta(\mathrm{G}))\rceil \leq \gamma(\mathrm{G})$.

That is, $\lceil\mathrm{p} / 5\rceil \leq \gamma(\mathrm{G})$. 
Case (i): $p=5 k+1, k \geq 1$.

In this case, $S=\left\{\mathrm{v}_{0}, \mathrm{v}_{5}, \mathrm{v}_{10}, \ldots, \mathrm{v}_{\mathrm{p}-9}, \mathrm{v}_{\mathrm{p}-4}\right\}$ is a dominating set of $\mathrm{G}$.

Hence, $\gamma(\mathrm{G}) \leq\lceil\mathrm{p} / 5\rceil$.

Case (ii): $n=5 k+2, k \geq 1$.

In this case, $\mathrm{S}=\left\{\mathrm{v}_{0}, \mathrm{v}_{5}, \mathrm{v}_{10}, \ldots, \mathrm{v}_{\mathrm{p}-10}, \mathrm{v}_{\mathrm{p}-5}\right\}$ is a dominating set of $\mathrm{G}$. Hence, $\gamma(\mathrm{G}) \leq\lceil\mathrm{p} / 5\rceil$.

Case (iii): $n=5 k+3, k \geq 1$.

In this case, $S=\left\{\mathrm{v}_{0}, \mathrm{v}_{5}, \mathrm{v}_{10}, \ldots, \mathrm{v}_{\mathrm{p}-6}, \mathrm{v}_{\mathrm{p}-1}\right\}$ is a dominating set of $\mathrm{G}$.

Hence, $\gamma(\mathrm{G}) \leq\lceil\mathrm{p} / 5\rceil$.

Case (iv): $n=5 k+4, k \geq 1$.

In this case, $\mathrm{S}=\left\{\mathrm{v}_{0}, \mathrm{v}_{5}, \mathrm{v}_{10}, \ldots, \mathrm{v}_{\mathrm{p}-7}, \mathrm{v}_{\mathrm{p}-2}\right\}$ is a dominating set of $\mathrm{G}$.

Hence, $\gamma(\mathrm{G}) \leq\lceil\mathrm{p} / 5\rceil$.

Case (v): $n=5 k+5, k \geq 1$.

In this case, $S=\left\{\mathrm{v}_{0}, \mathrm{v}_{5}, \mathrm{v}_{10}, \ldots, \mathrm{v}_{\mathrm{p}-8}, \mathrm{v}_{\mathrm{p}-3}\right\}$ is a dominating set of $\mathrm{G}$.

Hence, $\gamma(\mathrm{G}) \leq\lceil\mathrm{p} / 5\rceil$.

So in all the cases, $\gamma(\mathrm{G}) \leq\lceil\mathrm{p} / 5\rceil$.

From (1) and (2), $\gamma(\mathrm{G})=\lceil\mathrm{p} / 5\rceil$.

In all the above cases, $S$ is also a restrained dominating set of $G$. Therefore, $\gamma_{r}(G)=\gamma(G)$.

Lemma 2.2: Let $G$ be a connected graph. Let $u \in V(G)$ is eccentric to atmost $m$ vertices, then $\lceil\mathrm{p} /(1+\mathrm{m})\rceil \leq \gamma_{\mathrm{ed}}(\mathrm{G})$.

Proof: Let $S$ be a $\gamma_{\text {ed }}$-set of $G$. A vertex in $G$ is eccentric to atmost $m$ vertices.

$$
\text { Hence, }\lceil\mathrm{p} /(1+\mathrm{m})\rceil \leq \gamma_{\mathrm{ed}}(\mathrm{G}) \text {. }
$$

Theorem 2.1: Let $G=C_{p}\langle 1,2\rangle$ be a connected graph then for any integer $p \geq 6$, 


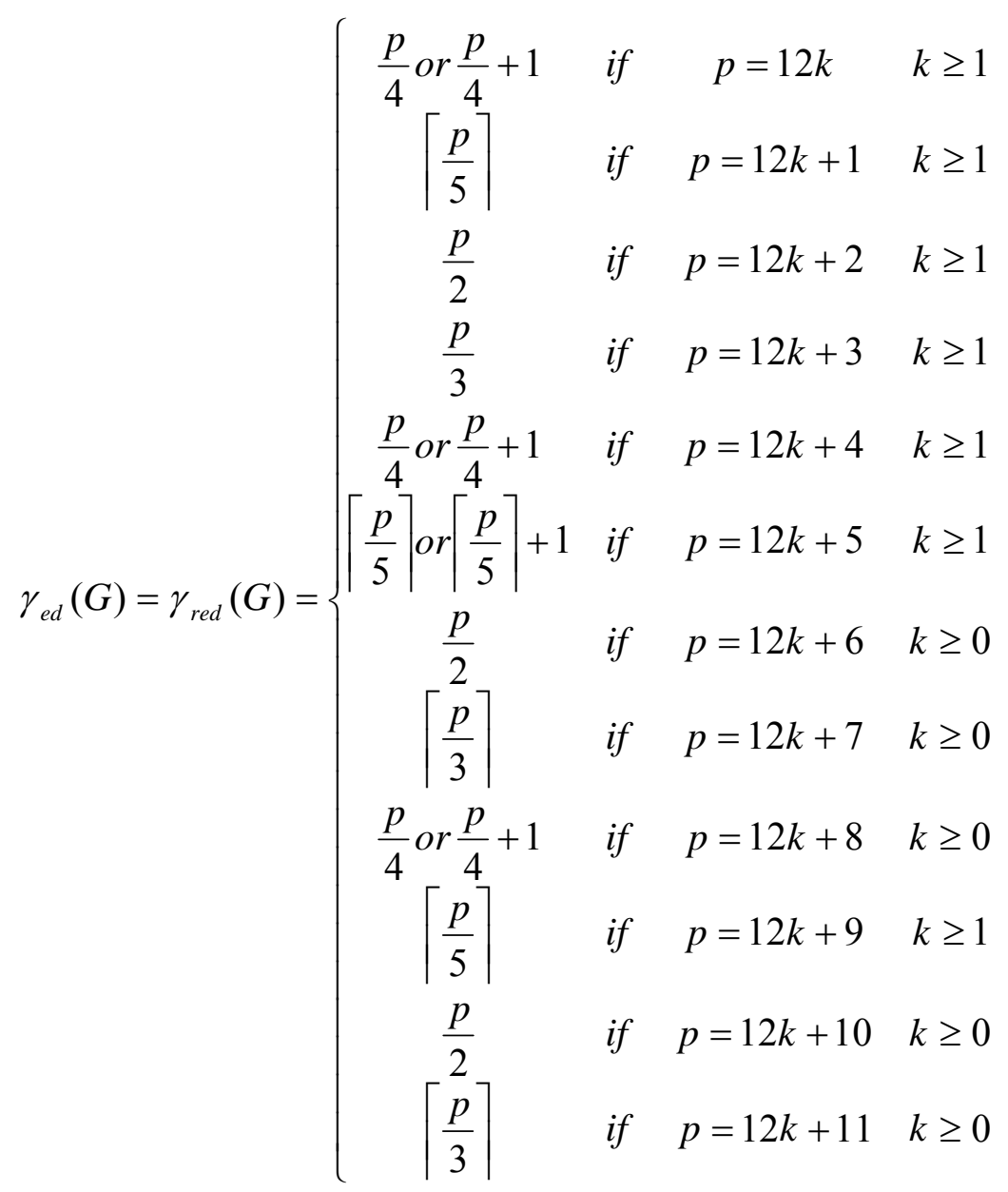

$\gamma_{\text {ed }}\left(C_{9}\langle 1,2\rangle\right)=3=\gamma_{\text {red }}\left(C_{9}\langle 1,2\rangle\right)$.

Proof: Let $\mathrm{G}=\mathrm{C}_{\mathrm{p}}\langle 1,2\rangle$ and let $\mathrm{v}_{0}, \mathrm{v}_{1}, \mathrm{v}_{2}, \ldots, \mathrm{v}_{\mathrm{p}-1}$ be the vertices of $\mathrm{G}$.

When $\mathrm{G}=\mathrm{C}_{9}\langle 1,2\rangle . \mathrm{G}$ is a 2 -self centered graph. The vertices $v_{\frac{p-3}{2}+i,}, \frac{v_{p-1}+i,}{2}, \frac{v_{p+1}+i,}{2}, \frac{v_{p+3}+i}{2}$ are the eccentric vertices of $\mathrm{v}_{\mathrm{i}}(\mathrm{i}=0,1,2, \ldots, \mathrm{p}-1) . \mathrm{S}=\left\{\mathrm{v}_{0}, \mathrm{v}_{4}, \mathrm{v}_{8}\right\}$ is a minimum eccentric dominating set of $\mathrm{G}$. $\mathrm{S}$ is also a minimum restrained eccentric dominating set of $G$. Thus, $\gamma_{\text {ed }}(G)=\gamma_{\text {red }}(G)=3$.

Case (i): $p=12 k, k \geq 1$

In this case, $G$ is a $\left(\frac{p}{4}\right)$-self centered graph. The vertices $v_{\frac{p-2}{2}+i,} v_{\frac{p}{2}+i,} v_{\frac{p+2}{2}+i}$ are the eccentric vertices of $v_{i}(i=0,1,2, \ldots, p-1)$. Therefore, $G$ is a 3 -eccentric point graph.

Hence, by Lemma $2.2, \frac{p}{4} \leq \gamma_{\text {ed }}(G)$. 
$\mathrm{S}=\left\{v_{0}, v_{4}, v_{8}, \ldots, v_{m}, v_{\frac{p+4}{2}}, v_{\frac{p+4}{2}+4}, \ldots, v_{t}\right\}$ is an eccentric dominating set of $\mathrm{G}$ (where $\mathrm{m}$ is the multiple of 4 such that $m$ is the largest integer less than or equal $p / 2$ and $t$ is of the form $\frac{p+4}{2}+4 n$ such that $\left.\mathrm{t} \leq \mathrm{p}-1\right) .|\mathrm{s}|=\frac{p}{4}$ if $\frac{p+4}{2}$ is a multiple of 4 , otherwise $|\mathrm{s}|=\frac{p}{4}$ +1 .

Thus, $\gamma_{\mathrm{ed}}(\mathrm{G})=\frac{p}{4}$, otherwise $\gamma_{\mathrm{ed}}(\mathrm{G})=\frac{p}{4}+1$.

From (1) and (2), $\gamma_{\text {ed }}(\mathrm{G})=\frac{p}{4}$ or $\frac{p}{4}+1$.

Case (ii): $p=12 k+1, k \geq 1$.

In this case, $\mathrm{G}$ is a $\left(\frac{p-1}{4}\right)$-self centered graph.

The vertices $\quad v_{\frac{p-3}{2}+i,} v_{\frac{p-1}{2}+i,} v_{\frac{p+1}{2}+i,} v_{\frac{p+3}{2}+i}$ are the eccentric vertices of $\mathrm{v}_{\mathrm{i}}(\mathrm{i}=0,1,2, \ldots, \mathrm{p}-1)$. Therefore, $\mathrm{G}$ is a 4 -eccentric point graph.

Hence, by Lemma 2.2, $[\mathrm{p} / 5] \leq \gamma_{\mathrm{ed}}(\mathrm{G})$.

$\mathrm{S}=\left\{v_{0}, v_{5}, v_{10}, \ldots, v_{m}, v_{\frac{p+5}{2}}, v_{\frac{p+5}{2}+5}, \ldots v_{t}\right\}$ is an eccentric dominating set of $\mathrm{G}$ (where $\mathrm{m}$ is the multiple of 5 such that $\mathrm{m}$ is the largest integer less than or equal $\mathrm{p} / 2$ and $\mathrm{t}$ is of the form $\frac{p+5}{2}+5 n$ such that $\left.\mathrm{t} \leq \mathrm{p}-1\right)$. $|\mathrm{S}|=\lceil\mathrm{p} / 5\rceil$.

Thus, $\gamma_{\mathrm{ed}}(\mathrm{G}) \leq\lceil\mathrm{p} / 5\rceil$.

From (3) and (4), $\gamma_{\text {ed }}(\mathrm{G})=\lceil\mathrm{p} / 5\rceil$.

Case (iii): $p=12 k+2, k \geq 1$.

In this case, $\mathrm{G}$ is a $\left(\frac{p+2}{4}\right)$-self centered graph.

The vertex $v_{\frac{p}{2}+i}$ is the eccentric vertex of $\mathrm{v}_{\mathrm{i}}(\mathrm{i}=0,1,2, \ldots, \mathrm{p}-1)$. Therefore, $\mathrm{G}$ is a self centered unique eccentric point graph.

Hence, $\gamma_{\text {ed }}(G) \geq \frac{p}{2}$.

$\mathrm{S}=\left\{\mathrm{v}_{0}, \mathrm{v}_{2}, \mathrm{v}_{4}, \ldots, \mathrm{v}_{\mathrm{p}-6}, \mathrm{v}_{\mathrm{p}-4}, \mathrm{v}_{\mathrm{p}-2}\right\}$ is an eccentric dominating set of $\mathrm{G}$.

Thus, $\gamma_{\mathrm{ed}}(\mathrm{G}) \leq \frac{p}{2}$.

From (5) and (6), $\gamma_{\text {ed }}(\mathrm{G})=\frac{p}{2}$. 


\section{Case (iv): $p=12 k+3, k \geq 1$}

In this case, $\mathrm{G}$ is a $\left(\frac{p+1}{4}\right)$-self centered graph. The vertices $v_{\frac{p-1}{2}+i}, \frac{v^{p+1}+i}{2}$ are the eccentric vertices of $v_{i}(i=0,1,2, \ldots, p-1)$. Therefore, $G$ is a 2 -eccentric point graph.

Hence, by Lemma 2.2, $\frac{p}{3} \leq \gamma_{\text {ed }}(\mathrm{G})$.

$\mathrm{S}=\left\{\mathrm{v}_{0}, \mathrm{v}_{3}, \mathrm{v}_{6}, \ldots, \mathrm{v}_{\mathrm{p}-9}, \mathrm{v}_{\mathrm{p}-6}, \mathrm{v}_{\mathrm{p}-3}\right\}$ is an eccentric dominating set of $\mathrm{G}$.

Thus, $\gamma_{\text {ed }}(\mathrm{G}) \leq \frac{p}{3}$.

From (7) and (8), $\gamma_{\mathrm{ed}}(\mathrm{G})=\frac{p}{3}$.

Case (v): $p=12 k+4, k \geq 1$.

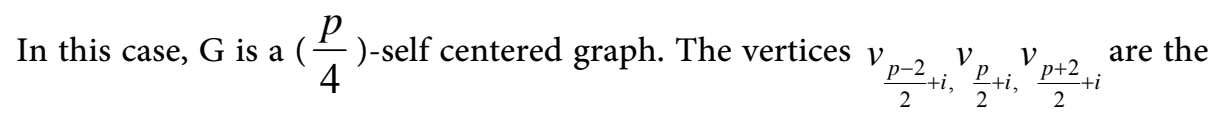
eccentric vertices of $v_{i}(i=0,1,2, \ldots, p-1)$. Therefore, $G$ is a 3 -eccentric point graph.

Hence, by Lemma $2.2, \frac{p}{4} \leq \gamma_{\mathrm{ed}}(\mathrm{G})$.

$\mathrm{S}=\left\{v_{0}, v_{4}, v_{8}, \ldots, v_{m}, v_{\frac{p+4}{2}}, v_{\frac{p+4}{2}+4}, \ldots, v_{t}\right\}$ is an eccentric dominating set of $\mathrm{G}$ (where $\mathrm{m}$ is the multiple of 4 such that $m$ is the largest integer less than or equal $p / 2$ and $t$ is of the form $\frac{p+4}{2}+4 n$ such that $\left.\mathrm{t} \leq \mathrm{p}-1\right)$. $|\mathrm{s}|=\frac{p}{4}$ if $\frac{p+4}{2}$ is a multiple of 4 , otherwise $|\mathrm{s}|=\frac{p}{4}+1$.

Thus, $\gamma_{\mathrm{ed}}(\mathrm{G})=\frac{p}{4}$, otherwise $\gamma_{\mathrm{ed}}(\mathrm{G})=\frac{p}{4}+1$.

From (9) and (10), $\gamma_{\mathrm{ed}}(\mathrm{G})=\frac{p}{4}$ or $\frac{p}{4}+1$.

Case (vi): $p=12 k+5, k \geq 1$.

In this case, $\mathrm{G}$ is a $\left(\frac{p-1}{4}\right)$-self centered graph.

The vertices $v_{\frac{p-3}{2}+i,} v_{\frac{p-1}{2}+i,} v_{\frac{p+1}{2}+i,} v_{\frac{p+3}{2}+i}$ are the eccentric vertices of $\quad \mathrm{v}_{\mathrm{i}}(\mathrm{i}=0$, $1,2, \ldots, p-1)$. Therefore, $G$ is a 4 -eccentric point graph.

Hence, by Lemma 2.2, $\lceil\mathrm{p} / 5\rceil \leq \gamma_{\mathrm{ed}}(\mathrm{G})$.

$\mathrm{S}=\left\{v_{0}, v_{5}, v_{10}, \ldots, v_{m}, v_{\frac{p+5}{2}}, v_{\frac{p+5}{2}+5}, \ldots, v_{t}\right\}$ is an eccentric dominating set of $\mathrm{G}$ (where $\mathrm{m}$ is the multiple of 5 such that $m$ is the largest integer less than or equal $p / 2$ and $t$ is of the 
form $\frac{p+5}{2}+5 n$ such that $\left.\mathrm{t} \leq \mathrm{p}-1\right)$. $|\mathrm{S}|=\lceil\mathrm{p} / 5\rceil$ if $\frac{p+5}{2}$ is a multiple of 5 , otherwise $|\mathrm{s}|=\lceil\mathrm{p} / 5\rceil+1$.

Thus, $\gamma_{\text {ed }}(G)=\lceil\mathrm{p} / 5\rceil$, otherwise $\gamma_{\text {ed }}(G)=\lceil\mathrm{p} / 5\rceil+1$

From (11) and (12), $\gamma_{\text {ed }}(G)=\lceil\mathrm{p} / 5\rceil$ or $\lceil\mathrm{p} / 5\rceil+1$.

Case (vii): $p=12 k+6, k \geq 0$.

In this case, $\mathrm{G}$ is a $\left(\frac{p+2}{4}\right)$-self centered graph. The vertex $v_{\frac{p}{2}+i}$ is the eccentric vertex of $v_{i}(i=0,1,2, \ldots, p-1)$. Therefore, $G$ is a self centered unique eccentric point graph.

Hence, $\gamma_{\text {ed }}(G) \geq \frac{p}{2}$.

$\mathrm{S}=\left\{\mathrm{v}_{0}, \mathrm{v}_{2}, \mathrm{v}_{4}, \ldots, \mathrm{v}_{\mathrm{p}-6}, \mathrm{v}_{\mathrm{p}-4}, \mathrm{v}_{\mathrm{p}-2}\right\}$ is an eccentric dominating set of $\mathrm{G}$.

Thus, $\gamma_{\text {ed }}(\mathrm{G}) \leq \frac{p}{2}$.

From (13) and (14), $\gamma_{\mathrm{ed}}(\mathrm{G})=\frac{p}{2}$

Case (viii): $\mathbf{p}=12 \mathrm{k}+7, \mathrm{k} \geq \mathbf{0}$.

In this case, $\mathrm{G}$ is a $\left(\frac{p+1}{4}\right)$-self centered graph. The vertices $v_{\frac{p-1}{2}+i, \frac{p+1}{2}+i}$ are the eccentric vertices of $\mathrm{v}_{\mathrm{i}}(\mathrm{i}=0,1,2, \ldots, \mathrm{p}-1)$. Therefore, $\mathrm{G}$ is a 2-eccentric point graph.

Hence, by Lemma $2.2,[\mathrm{p} / 3\rceil \leq \gamma_{\text {ed }}(\mathrm{G})$.

$\mathrm{S}=\left\{v_{0}, v_{3}, v_{6}, \ldots, v_{m}, v_{\frac{p+3}{2}}, v_{\frac{p+3}{2}+3}, \ldots v_{t}\right\}$ is an eccentric dominating set of $\mathrm{G}$ (where $\mathrm{m}$ is the multiple of 3 such that $m$ is the largest integer less than or equal to $p / 2$ and $t$ is of the form $\frac{p+3}{2}+3 n$ such that $\left.\mathrm{t} \leq \mathrm{p}-1\right)$. $|\mathrm{S}|=\lceil\mathrm{p} / 3\rceil$.

Thus, $\gamma_{\mathrm{ed}}(\mathrm{G}) \leq\lceil\mathrm{p} / 3\rceil$.

From (15) and (16), $\gamma_{\text {ed }}(G)=\lceil\mathrm{p} / 3\rceil$

Case (ix): $p=12 k+8, k \geq 0$.

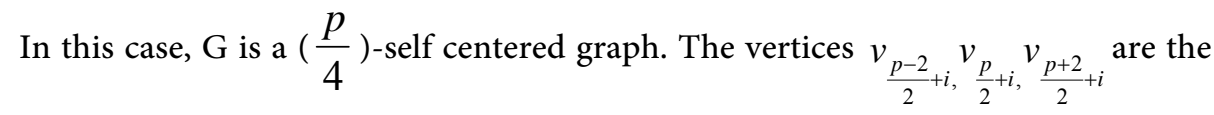
eccentric vertices of $v_{i}(i=0,1,2, \ldots, p-1)$. Therefore, $G$ is a 3-eccentric point graph. 
Hence, by Lemma $2.2, \frac{p}{4} \leq \gamma_{\text {ed }}(\mathrm{G})$.

$\mathrm{S}=\left\{v_{0}, v_{4}, v_{8}, \ldots, v_{m}, v_{\frac{p+4}{2}}, v_{\frac{p+4}{2}+4}, \ldots, v_{t}\right\}$ is an eccentric dominating set of $\mathrm{G}$ (where $\mathrm{m}$ is the multiple of 4 such that $m$ is the largest integer less than or equal $p / 2$ and $t$ is of the form $\frac{p+4}{2}+4 n$ such that $\left.\mathrm{t} \leq \mathrm{p}-1\right) .|\mathrm{s}|=\frac{p}{4}$ if $\frac{p+4}{2}$ is a multiple of 4 , otherwise $|\mathrm{s}|=\frac{p}{4}+1$.

Thus, $\gamma_{\text {ed }}(\mathrm{G})=\frac{p}{4}$, otherwise $\gamma_{\text {ed }}(\mathrm{G})=\frac{p}{4}+1$.

From (17) and (18), $\gamma_{\text {ed }}(\mathrm{G})=\frac{p}{4}$ or $\frac{p}{4}+1$

Case $(x): p=12 k+9, k \geq 1$.

In this case, $\mathrm{G}$ is a $\left(\frac{p-1}{4}\right)$-self centered graph.

The vertices $v_{\frac{p-3}{2}+i,} v_{\frac{p-1}{2}+i,} v_{\frac{p+1}{2}+i}, \frac{v_{p+3}}{2}+i$ are the eccentric vertices of $\mathrm{v}_{\mathrm{i}}(\mathrm{i}=0,1,2, \ldots, \mathrm{p}-1)$. Therefore, $\mathrm{G}$ is a 4-eccentric point graph.

Hence, by Lemma $2.2,\lceil\mathrm{p} / 5\rceil \leq \gamma_{\text {ed }}(\mathrm{G})$.

$\mathrm{S}=\left\{v_{0}, v_{5}, v_{10}, \ldots, v_{m}, v_{\frac{p+5}{2}}, v_{\frac{p+5}{2}+5}, \ldots v_{t}\right\}$ is an eccentric dominating set of $\mathrm{G}$ (where $\mathrm{m}$ is the multiple of 5 such that $\mathrm{m}$ is the largest integer less than or equal to $\mathrm{p} / 2$ and $\mathrm{t}$ is of the form $\frac{p+5}{2}+5 n$ such that $\left.\mathrm{t} \leq \mathrm{p}-1\right) .|\mathrm{S}|=\lceil\mathrm{p} / 5\rceil$.

Thus, $\gamma_{\text {ed }}(\mathrm{G}) \leq\lceil\mathrm{p} / 5\rceil$.

From (19) and (20), $\gamma_{\text {ed }}(G)=\lceil\mathrm{p} / 5\rceil$.

Case (xi): $p=12 k+10, k \geq 0$.

In this case, $\mathrm{G}$ is a $\left(\frac{p+2}{4}\right)$-self centered graph.

The vertex $v_{\frac{p}{2}+i}$ is the eccentric vertex of $\mathrm{v}_{\mathrm{i}}(\mathrm{i}=0,1,2, \ldots, \mathrm{p}-1)$. Therefore, $\mathrm{G}$ is a self centered unique eccentric point graph.

Hence, $\gamma_{\text {ed }}(G) \geq \frac{p}{2}$.

$\mathrm{S}=\left\{\mathrm{v}_{0}, \mathrm{v}_{2}, \mathrm{v}_{4}, \ldots, \mathrm{v}_{\mathrm{p}-6}, \mathrm{v}_{\mathrm{p}-4}, \mathrm{v}_{\mathrm{p}-2}\right\}$ is an eccentric dominating set of $\mathrm{G}$.

Thus, $\gamma_{\text {ed }}(\mathrm{G}) \leq \frac{p}{2}$. 
From (21) and (22), $\gamma_{\mathrm{ed}}(\mathrm{G})=\frac{p}{2}$.

Case (xii): $p=12 k+11, k \geq 0$

In this case, $\mathrm{G}$ is a $\left(\frac{p+1}{4}\right)$-self centered graph.

The vertices $v_{\frac{p-1}{2}+i,} v_{\frac{p+1}{2}+i}$ are the eccentric vertices of $\mathrm{v}_{\mathrm{i}}(\mathrm{i}=0,1,2, \ldots, \mathrm{p}-1)$. Therefore, $G$ is a 2-eccentric point graph.

Hence, by Lemma 2.2, $\lceil\mathrm{p} / 3\rceil \leq \gamma_{\mathrm{ed}}(\mathrm{G})$.

$\mathrm{S}=\left\{v_{0}, v_{3}, v_{6}, \ldots, v_{m}, v_{\frac{p+3}{2}}, v_{\frac{p+3}{2}+3}, \ldots v_{t}\right\}$ is an eccentric dominating set of $\mathrm{G}$ (where $\mathrm{m}$ is the multiple of 3 such that $m$ is the largest integer less than or equal $p / 2$ and $t$ is of the form $\frac{p+3}{2}+3 n$ such that $\left.\mathrm{t} \leq \mathrm{p}-1\right)$. $|\mathrm{s}|=\lceil\mathrm{p} / 3\rceil$.

$$
\text { Thus, } \gamma_{\mathrm{ed}}(\mathrm{G}) \leq\lceil\mathrm{p} / 3\rceil \text {. }
$$

From (23) and (24), $\gamma_{\text {ed }}(\mathrm{G})=\lceil\mathrm{p} / 3\rceil$.

In all the above cases, $\mathrm{S}$ is also a restrained eccentric dominating set of $\mathrm{G}$.

Therefore, $\gamma_{\text {red }}(G)=\gamma_{\text {ed }}(G)$.

\section{Conclusion:}

Here we have studied eccentric domination and restrained eccentric domination in circulant graph $C_{p}\langle 1,2\rangle$, and also studied a bound for the eccentric domination number of a connected graph.

\section{References:}

[1] Bhanumathi, M., John Flavia, J., and Kavitha, M., Restrained Eccentric Domination in Graphs, International Journal of Engineering Science, Advanced Computing and Bio-Technology, Vol.5, No.1, January-March 2014, pp. 24-36.

[2] Bhanumathi, M., John Flavia, J., Number of Minimum Eccentric Dominating sets in paths, International Journal of Engineering Science, Advanced Computing and Bio-Technology, Vol.6, No.4, October-December 2015, pp. 114-118.

[3] Buckley, F., Harary, F., Distance in graphs, Addison-Wesley, Publishing company (1990).

[4] Cockayne, E.J., Hedetniemi, S.T., Towards a theory of domination in graphs \& Networks, 7:247-261, 1977.

[5] Harary, F., Graph theory, Addition-Wesley Publishing Company Reading, Mass (1972).

[6] Janakiraman, T.N., Bhanumathi, M., Muthammai, S., Eccentric domination in graphs, International Journal of Engineering science, Advanced Computing and Bio-Technology, Volume 1, No.2, pp 1-16, 2010. 
[7] NadarJafari Rad, Domination in Circulant Graphs, An. St. Univ. Ovidius Constanta, Vol. 17(1), 2009, 169-176.

[8] Teresa W. Haynes, Stephen T. Hedetniemi, Peter J. Slater, Fundamentals of Domination in graphs, Marcel Dekkar, New Yark(1998).

\section{Authors' Profile:}

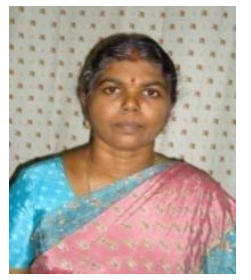

M. Bhanumathi was born in Nagercoil, Tamilnadu, India in 1960. She received her B.Sc., M.Sc. and M.Phil. degrees in Mathematics from Madurai Kamaraj University, India in 1981, 1983 and 1985 respectively. In 1987, she joined as Assistant Professor of Mathematics in M.V.M. Govt. Arts College for Women, Dindigul affiliated to Madurai Kamaraj University, India. Since 1990, she has been with the PG department of Mathematics in Government Arts College for Women, Pudukkottai. She did her research under Dr.T.N.Janakiraman at National Institute of Technology, Trichy for her doctoral degree, and received her Ph.D degree from Bharathidasan University in 2005. She became Reader in 2005 and Associate Professor in 2009. Her current research interests in Graph Theory include Domination in Graphs, Graph Operations, Distance in Graphs, Decomposition of Graphs, Metric dimension and Topological indices of graphs. She has published more than 70 research papers in national/international journals. She is currently the Principal in Government Arts and Science College, Kadaladi, Ramanathapuram district, Tamilnadu, India.

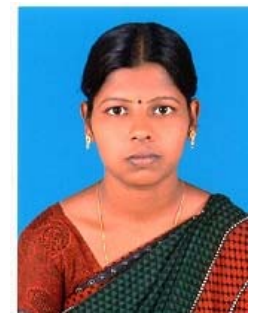

research papers.

R. Niroja was born in Thulukkaviduthy, Thanjavur district, Tamilnadu, India in 1989. She received her B.Sc and M.Sc degree in Mathematics from Bharathidasan University, Trichy in 2010 and 2012 and received B.Ed degree in Mathematics from Tamilnadu Tearchers Education University, Chennai in 2013. She received M.Phil degree in Mathematics from Bharathidasan University, Trichy in 2014 respectively. She is pursuing research in the Department of Mathematics, Government Arts College for Women (Autonomous), Pudukkottai, Tamilnadu, India. Her area of interest is Graph Theory. She has published 3 